\title{
Issues and Outlook
}

\author{
NANCY C. MAKI, M.D., DANIEL WALDEN, \\ and LAWRENCE COHEN, M.S.W.
}

\section{Medicare's Effects on Medical Care}

$\mathrm{U}$ TILIZATION review as we know it today has developed from two basic processes in evaluating the provision of medical care which have achieved increasing importance over the past three decades (1). These two processesnow merged by Congress in Medicare-are medical audit, focused on improving the quality of medical care, and control of bed utilization, focused on decreasing capital and operating costs and emptying scarce hospital beds.

Although rising costs and scarcity of beds have exerted a major influence on the development of utilization review, the earlier and overriding influence on this concept has been the physician's pursuit of excellence reflected in part in medical audit. The rapid growth of organizations such as the Professional Activities StudyMedical Audit Program (PAS-MAP) and the Hospital Utilization Project reflects the growing interest of hospital medical staffs in utilization review.

Drawing on the experience of an experiment begun in 1950 in centralized collection and processing of data on hospital medical care, the Commission on Professional and Hospital Activities was created in 1956. Under PAS-MAP

Dr. Maki is chief, Planning and Development Section, Mr. Walden is a health services officer, and Mr. Cohen was a health services officer, all in the Policy and Standards Branch, Division of Medical Care Administration, Public Health Service. the collection, tabulation, and analysis of information for improving medical practices and the administration of hospitals have been developed to aid hospital medical staffs in evaluating the quality of their own patient care. Frequent reports of basic patient data, admission findings, investigation, treatment, and results with indices of quality and quantity of medical care are prepared by the commission.

In addition to providing informational services, the Hospital Utilization Project in Pennsylvania furnishes frequent onsite consultation to hospital medical staffs regarding their utilization review activities. These visits are made by a physician, medical record librarian, or nurse consultant, depending on the particular needs of the facility.

The benefits from physicians' medical audit activities in the 20 years preceding Medicare predestined widespread application of a mechanism for appraising quality in the medical care system, with or without the passage of new health legislation. 'The American Medical Association's House of Delegates, while recognizing the interest of other groups, accepted major responsibility for reviewing utilization and quality controls in 1964. At this time, the American Medical Association also recommended that "State and local medical societies should urge and assist medical staffs of hospitals to form hospital utilization committees." The American Medical Association sponsored its first national conference on utilization review in November 1965 and its second one in November 1967.

The second historic root of utilization review, 
controlling the use of hospital beds, is characterized by concern about the rapidly rising demand for hospitalization and increasing costs of care. Use of hospitals has received growing interest and attention by Blue Cross as well as the Federal Government and the medical profession. The interest of Blue Cross has been related primarily to rising costs of hospital care which has led to higher premiums imposed on subscribers. 'The Blue Cross Association has encouraged utilization review through publications and sponsorship of professional meetings. Certain individual Blue Cross plans have required institutions to conduct review in order to be eligible for reimbursement.

\section{Utilization Review Under Medicare}

In the development of legislation establishing health insurance for the aged, both historic roots of utilization review were considered in an effort to insure quality and minimize cost. Under Medicare an acceptable utilization review plan focuses on quality by providing for the review, on a sample or other basis, of admissions, duration of stays, and professional services furnished. Such a plan focuses on cost by providing for review of each case of continuous extended duration. A single committee may deal with both aspects of utilization review or the functions may be divided among two or more committees.

The Medicare program requires that all participating hospitals and extended care facilities have in effect a plan for utilization review which applies at least to the services to patients entitled to benefits under the law. Since experience in utilization review had been fragmented and localized before Medicare, the regulations implementing the law were designed to encourage flexibility and experimentation in the structure and function of the utilization review committee.

At the outset of the Medicare program, hospitals and extended care facilities applying for certification submitted written plans describing the intended functioning of their utilization review programs. Because most institutions did not have functioning utilization review committees before July 1, 1966, these plans were accepted as evidence of conformity with the statutory requirements.

Ender the law designated State agencies are responsible for making periodic recommendations to the Social Security Administration as to whether hospitals, extended care facilities, and other providers should be certified for continued participation in the program. State agencies are responsible also for giving consultation to providers concerning any lack of compliance with the statutory and regulatory requirements.

The State agencies now have begun the recertification process which will include an appraisal of the actual operation of utilization review committees. If at the time of resurvey the State agency determines that the hospital or extended care facility does not have a functioning utilization review committee which meets the minimum requirements of the law, the institution may be denied recertification.

Fiscal intermediaries also have been given responsibility to assist hospitals and extended care facilities in the following aspects of utilization review.

1. Identifying and evaluating factors which impair effective utilization review.

2. Assisting in developing methods for collecting data and analyzing utilization review activities.

Preliminary data on Medicare show great variations in approaches to and competence in carrying out the review process. A number of hospitals, extended care facilities, and communities have developed innovative approaches to utilization review, while others have initiated plans designed primarily to meet the minimum requirements of the law.

A preliminary study of the 6,738 hospitals certified at the beginning of the program indicates that approximately 97 percent of these institutions have a utilization review committee sponsored by the hospital itself. Utilization review committee sponsors for the remaining 3 percent include local medical societies, two or more hospitals, and community organizations other than medical societies.

While range in the size of the committees was substantial, 43 percent have three to five members and 17 percent have six or seven members. Approximately 39 percent of the hospitals have persons who are not physicians on the committee. Hospital administrators serve on 35 percent of the committees, medical record librari- 
ans on 14 percent, nurses on 10 percent, and social workers on 3 percent.

Since a definition of extended duration was not established in the law or in the regulations, the committees were free to develop their own, and there is considerable range among them. Approximately 28 percent of the hospital committees have defined extended stay as 30 days or more, 25 percent have chosen between 20 and 24 days, and 14 percent have definitions which vary by the diagnostic category of the patient.

\section{The Community-Based Approaches}

The various approaches developed in community-based utilization review plans can be grouped into two general classifications. First, some plans provide a central mechanism for electronic processing of data for central anlyses of general trends and, in certain cases, professional and technical consultation to individual utilization review committees. However, the actual utilization review is undertaken by the medical staff of each facility. The Hospital Utilization Project in western Pennsylvania and the Professional Activity Study in Ann Arbor, Mich., are two well-known examples of the central mechanism approach.

The second approach includes not only a central mechanism for processing and analyzing data and for consultation, but it also carries out the actual utilization review in each participating facility. This community approach to utilization review has been successful in regional plans for extended care facilities and in plans for hospitals and extended care facilities in rural areas.

When it is impractical to develop an institution-based committee because a provider is too small or does not have an organized medical staff, the Medicare legislation requires that the utilization review committee be composed as a group outside the institution, that is, the second approach. This group may be established by either the local medical society with the participation of some or all providers within the area or any other appropriate means which has the approval of the Secretary of Health, Education, and Welfare.

Obviously a community-based utilization review plan has special advantages in promulgating uniformly high standards of care for institutions within the community, identifying needed community health services, and in assuring that all of the health resources of the community are maximally useful to the population. Community-based utilization review plans also encourage interinstitutional relationships and exchanges of information with resulting benefits to the individual patient and the community. Such arrangements, for example, may make it possible for the patient to be transferred promptly from one facility to another without interruption of his treatment as his needs change.

The development of community-based utilization review plans has been encouraged by the Public Health Service because the most effective utilization review considers the total health resources of a community in contrast to focusing on a single institution. When health agencies are integrated so that each unit of the health system is specializing in the services it is best equipped to provide, the total system is more effective and efficient.

The Public Health Service currently is sponsoring a number of utilization review projects through the contract mechanism. Some of these projects are described in this issue. This support of plans in various settings includes financial assistance for several community-based plans covering extended care facilities in urban areas and for a hospital and extended care facility plan in a sparsely populated State. The development of model psychiatric utilization review plans is also being supported as are studies for developing and testing various strategies for conducting utilization review.

\section{Assumptions About Utilization Review}

Since 1950 studies on assuring high-quality medical care show a great deal of unanimity regarding the objectives, criteria, and means of implementing the medical audit component of utilization review. The differences are primarily concerned with depth and detail.

Generally accepted approaches to the measurement of quality of medical care include the following.

1. Measurement of performance through the use of criteria for the management of various diagnostic categories. 
2. Studies on appropriateness and extent of diagnostic procedures.

3. Studies on timely and proper use of consultations.

4. Determination of justification for surgery.

5. Review of complications and deaths.

6. Comparison of data derived from other hospitals of merit.

Most investigators agree that effective utilization review, particularly the medical audit component, can be done by analyzing clinical records, with emphasis on establishing valid criteria and standards for accurately measuring the quality of care. There has been general support for review on a continuing basis with concentration on one category at a time-such as a selected diagnosis or groups of similar diagnoses, selected operations, all deaths, transfusions, or prenatal care. The consensus is that utilization review can elevate the quality of medical care best through education and that a program of utilization review can be planned and implemented best by physicians.

\section{A Means of Solving Medical Care Problems}

Utilization review may help to solve some of the problems in medical care attributed to the following.

1. Growing demands for better and more comprehensive medical care exacted by an increasingly sophisticated, educated, and healthconscious public.

2. Continuing barriers to adequate health care for segments of the population, especially the poor.

3. Rapidly rising medical care costs.

4. The time lag between the discovery of new medical knowledge and applying it to the population. Techniques to insure optimal delivery of health care have not kept pace with the remarkable discoveries which have come as a result of research in medicine.

5. Inadequate techniques to assist physicians in updating their medical knowledge.

6. Less than maximal productivity of physicians. For example, other health personnel can perform tasks which, though commonly performed by physicians, do not require the amount of education and experience which a physician possesses.

While optimal solutions to many medical care problems will result from changes in the organization and financing of health services, these changes are not likely to occur quickly. In the meantime, utilization review can lessen some of the problems. As a system which can incorporate constant updating of criteria for the care or management of patients, utilization review can facilitate more rapid dissemination of new and important knowledge to all physicians. By emphasizing the most appropriate level of care for each patient, utilization review committees can help reduce total cost of medical care. Increased public awareness of the philosophy and purpose of utilization review may encourage more rational and more effective demands on the medical care system.

Information derived from the utilization review process can be used to plan more productivity of scarce health manpower, construction of health care facilities, and location of medical equipment to provide optimal service to the population.

\section{Barriers to Effective Use}

Because of the limited scope of the Medicare program, its legal requirements for utilization review were not and could not be designed to ensure realization of the maximum potential of the process. The legislation does not require institutions to relate their utilization review activities to the community or to use the entire patient population as a pool from which to draw cases for review.

Problems among professionals. Many other widespread obstacles to effective use of utilization review have been overcome in areas where physicians have had extensive experience with the process. The remaining barriers relate in part to attitudes among certain physicians, administrators, and other allied health professionals. For example, it is commonly agreed that effective utilization review depends on consensus within the committee as to what constitutes quality care. This consensus can be reached best by using established standards or definitions.

However, some physicians visualize utilization review as an attempt to limit the individuality of the practitioner and to initiate rigidly standardized or "cookbook style" medicine. These critics insist that the criteria or standards 
which are the bases for utilization review cannot be delineated without eliminating the professional judgment fundamental to medical practice.

The reverse could be true if utilization review were really effective. Standards or criteria ideally should be open to revision so that as new knowledge becomes available, they may become a mechanism for communicating to all physicians the most up-to-date methods of practice.

Some professionals contend that even with the use of established standards, utilization review merely assesses the quality of medical records rather than the quality of care. In other words, evaluation of care based on the medical record relies on the completeness and accuracy of the record. There may be a discrepancy between care recorded and care given, however. The answer, in part, suggests more comprehensive medical records.

Another barrier to effective utilization review relates to its newness. Many physicians and administrators do not have an adequate concept of its purpose and process. Many physicians and other professional staff, who serve on utilization review committees, need to understand and fully utilize the techniques and processes.

The low level of competence in utilization review can be attributed in part to failure to integrate concepts concerning physicians' responsibilities pertaining to utilization review into the medical school curriculum and to unavailability of teaching materials.

Moreover, in some medical specialties, particularly psychiatry, little effort has been directed to modifying the concept of utilization review so that it will be a useful tool in that particular specialty. The American Psychiatric Association, with support from the Public Health Service, has now begun to develop objectives and models for psychiatric utilization review and to identify special problems which may impede this type of review.

When medical schools and professional organizations develop curriculums and when most physicians accept the value of utilization review, then professional competence will be developed to insure realization of the full potentials of the process. However, until the barrier of physician resistance is overcome, there will be no major advancement in competence for utilization review.

Interdisciplinary relationships. A barrier to the effectiveness and efficiency of utilization review is rejection by physicians of the function of administrators, nurses, social workers, medical record librarians, and other nonphysician health professionals on utilization review committees. One of the major functions of the utilization review committee is to suggest administrative changes, and inclusion of administrators and other professionals on the utilization review committee would greatly increase communication between the committee and other personnel so that change can be planned and implemented.

Nurses can contribute to the utilization review process because they are familiar with the patients' needs, attitudes, and functional status. Social workers can contribute to utilization review by alerting the physicians to social problems which may affect patients' well-being and help in planning their discharges. The medical record librarian can routinely assist the utilization review committee in many areas relating to medical records.

The patient and the family. Another factor limiting the effectiveness of utilization review is that use of hospitals and extended care facilities is not dependent solely on the patient's physical condition. The utilization review committee frequently is confronted with socioeconomic problems of the patient and his family. Perhaps the most common one affecting the patient's health and well-being is his concern for the payment of medical expense. Persons needing medical care may request hospitalization because their health insurance covers inpatient services more fully than outpatient care. Families may urge physicians to hospitalize relatives because for the family, this may be the cheapest and most convenient method of guaranteeing that the patient's health and dependency needs are met.

This approach relieves family members of personally providing care. In such situations the physician may be influenced by the patient or his family. At times the utilization review committee may be able to reinforce the attending physician's resolve since he can tell the patient and his family that the committee will not 
or does not find further stay medically necessary. However, in other cases, especially when there are complex emotional or socioeconomic problems or both, the committee may have difficulty in acting decisively.

The public should be made aware of the philosophy and purpose of utilization review in assuring quality services and cost savings. Then individual patients and families may accept decisions concerning the level of care needed and the setting in which it should be provided. Public education could eliminate some of the pressure exerted on physicians.

\section{Summary and Conclusions}

Utilization review holds definite promise as one tool which can be used to help the serious medical care problems in the next decade. Community-based utilization review has five major potentials or objectives.

1. Insure high-quality medical care to the population.

2. Reduce the total cost of medical care within the community.

3. Provide a mechanism for improving administrative policies and procedures for individual institutions and between institutions.

t. Provide a mechanism for the continuing medical education of physicians through participation in formulating and using standards of care for various disease states and through the increased exchange of information among peers.

5. Derelop and analyze patient care profiles and other data which can be fed into a community or regional health planning mechanism and thus be used for appraising and planning community health resources.

Apparently there is a great need for further demonstration or experimentation in the various approaches to utilization review with special emphasis on those methods which are aimed at (a) community objectives in the area of health resources plamning and use and $(b)$ continuing education for physicians. 'The Public Health Service is currently working with a group of consultants to design a coordinated program of research in utilization review, since experimentation and research are needed to test the effectiveness and efficiency of various approaches to utilization review.

\section{REFERENCE}

(1) U.S. Public Health Service: Utilization review. A selected bibliography, 1933-1967. Division of Medical Care Administration, Arlington, Va., 1968 .

\section{Intercommunication Among Health Facilities}

Someday the nation's hospitals and other medical facilities will communicate with each other via a vast computer network which will provide general and emergency health information at the flip of a switch or the turn of a dial.

The Public Health Service has undertaken a study to establish guidelines and to develop standards criteria to insure the ability for intercommunication among health facilities, regardless of the type of internal computer system used.

The first phase of this long-range project, a joint effort of the Service's Division of Hospital and Medical Facilities and the National Bureau of Standards, will be underwritten by the division at a cost of $\$ 95,000$ and will cover a 9-month period.

During the first phase the two agencies will delineate present problems, identify and classify the kinds of information to be communicated, study the types of equipment and development efforts being applied in this area, and become familiar with past and present research activity. Particular attention will be given to privacy of data, validation of patient data, message formats, and communication protocol. Error recovery and control procedures will also be investigated.

If intercommunication systems are to develop in an orderly manner, early steps are important to determine the identity and classification of information to be communicated among health facilities. For the greatest benefit to patient care, it is also essential that the requirements of the medical community be made known to the information processing industry to insure that these requirements be included in the planning of the intercommunication network. 